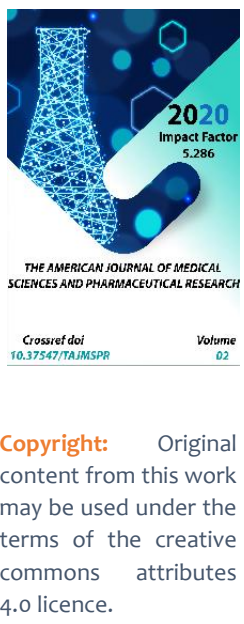

4.0 licence.

\section{Course Of Post-Infarction Depression Taking Into Account The Time Of Its Once}

\author{
Mukhtorova H.K. \\ Bukhara State Medical Institute; Department Of Psychiatry, Narcology And Medical \\ Psychology; Bukhara, Uzbekistan \\ Mukhamadieva N.B. \\ Bukhara State Medical Institute; Department Of Psychiatry, Narcology And Medical \\ Psychology; Bukhara, Uzbekistan \\ Rustamov U.T. \\ Bukhara State Medical Institute; Department Of Psychiatry, Narcology And Medical \\ Psychology; Bukhara, Uzbekistan
}

\title{
ABSTRACT
}

A study of 341 patients with an established diagnosis of acute myocardial infarction was carried out. The patients are divided into 2 groups. The first, main, included 233 patients with acute myocardial infarction, who subsequently developed depressive disorders, confirmed clinically and using diagnostic scales; the second group consisted of 108 patients who also had acute myocardial infarction, but did not subsequently suffer from symptoms of depression. Clinical and dynamic observation of patients in the postinfarction period was carried out with control of the condition in a month, three months, six months, 12 months after myocardial infarction. Clinical observation was used to detect the presence of symptoms of depression. Among patients with MI with DS in the first days after $\mathrm{MI}$, more than half $(51.1 \%)$ reported a permanent decrease in mood more often than other symptoms, i.e. these patients showed an affective component of depression, while the ideator component of depression, which manifested itself in thinking retardation to one degree or another, was in $27.5 \%$ of patients, motor retardation (motor component) was detected in $21.5 \%$ of those observed. In patients with almost the same frequency, the anxious and melancholic type of affect was noted ( $47.0 \%$ and $41.2 \%$, respectively), the dysphoric type of affect was found in $11.8 \%$ of cases, which is 4 times less than anxious and 3.5 times less than the melancholic type. In cases of prolonged depression, the severity of affective disorders more often directly correlated with the severity of the physical condition. If not so long-term depressive episodes were largely due to "their own vision of the disease", the severity of their symptoms depended on the conversation with the doctor and the information received from him, the degree of awareness of his diagnosis, possible complications, and not very much depended on the general somatic status itself. then the course of prolonged depression in patients worsened with the aggravation of the cardiological and general somatic condition of patients. Psychosomatic parallelism in the majority of protracted depressions was manifested by the generalization of asthenic symptom complexes (increased general weakness, intolerance to exertion, lethargy, adynamia, severe daytime sleepiness in combination with early insomnia) with deterioration 
of the somatic state. At the same time, it was noted that with prolonged depression, there were always more or less pronounced cognitive disorders (reduced memory for past events, limited ability to comprehend what was happening around, remember new information, impaired concentration). Postinfarction depressive episodes lasting up to six months can be attributed to nosogenies; depressive episodes of postinfarction genesis with a protracted course, probably with a high degree of confidence can be attributed to somatogenias.

\section{KEYWORDS}

Postinfarction depression, comorbidity, risk factors, pathogenetic predictors, anxious and melancholic depression.

\section{INTRODUCTION}

Changes in mood (affect, emotions) permeate the entire biopsycho-socio-spiritual essence of a person in health and disease. However, ICD10 treats affective disorders from three somewhat conflicting approaches. They are included both in the framework of organic affective disorder (Fo6.3) and in the framework of anxiety and adjustment disorders (F40-43), and the basic ones are affective disorders (F 30-39), where psychogenic depressions of varying degrees have "dissolved" among the endogenous ones. gravity. In European and Russian psychiatry, predominantly of the twentieth century, the etiological division was, in particular, the classification of depressions by K. Schneider into organic, endogenous and psychogenic. As the twentieth century was a century of depressions, so in the twenty-first century an even greater increase in the number of depressive states is predicted, and above all of a psychogenic nature due to an increase in the number of not only and not so much natural and man-made disasters, but due to the global financial recession ("depression") social and mental terrorism, globalization, unemployment, destruction of the institution of the family and community, personal and professional dissatisfaction, which are reflected in the ideas about the quality of life in society, the country, the macro- and microsociety, the individual itself and his understanding of happiness and fullness of life. All this leads to a discrepancy between external and internal representations in interpersonal and intrapersonal relationships, accompanied by a decrease in mood as a natural reaction of the body to biological, psychological, social or spiritual needs of a person with a desire to overcome these obstacles (Nosachev I.G. et al., 2017) $[6,11,12]$.

Depression is often comorbid with other, somatic, diseases, and in such cases, diseases of two different spheres - mental and somatic aggravate each other, sometimes leading to serious consequences. Anxiety and depression are not static conditions, and neither is dominant, although both are pronounced. It is only characteristic that against the background of depression, anxiety intensifies and takes on a huge scale. Each of these conditions enhances the effect of another syndrome, and 
the presence of an underlying endogenous disease is a factor contributing to the occurrence of anxiety and depressive states in patients. It is obvious that anxiety and depression are satellites of endogenous mental disorders, which in one case manifest themselves dichotomously, and in another integratively. The paradigm of views on the etiopathogenesis of affective states in endogenous mental disorders indicates that this issue is far from a final solution and requires a new formulation of problems and their solution. Among patients with cardiovascular diseases, the incidence of concomitant depression is $22-33 \%$. In $17-27 \%$ of patients with coronary artery disease undergoing coronary angiography, depression is detected, and in patients in the postinfarction period, depression is found in $16-45 \%$ of cases. The presence of depression in patients with cardiovascular diseases not only complicates the course and therapy of these disorders, but also shortens the life expectancy of patients. It is clear and predictable that the most pronounced emotional disorders are observed in patients who have had myocardial infarction, since even with a satisfactory state of health, the diagnosis of myocardial infarction is associated in a person with a threat to life $[7,8,9,10]$. Along with worrisome fears about health, there are dark thoughts about the future, depression, fear of possible disability, disturbing thoughts about the wellbeing of the family. Without appropriate intervention, these disorders are fixed and persist for one year in $25 \%$ of survivors. According to other data, mental disorders were diagnosed in $28 \%$ of cases. In $50 \%$ of patients an intensification of neurotic features was observed (Drobizhev M.Yu., 2003, Krasnov V.N., 2001, Smulevich A.B., 2001, Dickens CM,
McGowan I., Percival C. et al., 2004, Beckers T .., 2001).

\section{MATERIALS AND RESEARCH METHODS}

A study of 341 patients with an established diagnosis of acute myocardial infarction was carried out. The patients are divided into 2 groups. The first, main, included 233 patients with acute myocardial infarction, who subsequently developed depressive disorders, confirmed clinically and using diagnostic scales; the second group consisted of 108 patients who also had acute myocardial infarction, but did not subsequently suffer from symptoms of depression. Clinical and dynamic observation of patients in the postinfarction period was carried out with control of the condition in a month, three months, six months, 12 months after myocardial infarction. To identify the presence of symptoms of depression, the method of clinical observation was used (from the first hours after the patient was admitted to the hospital with a diagnosis of acute myocardial infarction), and the Hamilton and Montgomery-Asberg scales were used during the first three days of hospital stay. If no depressive symptoms were found on the first day after an ischemic attack, then for diagnostic purposes, the scales were used in a hospital setting daily until clinically significant symptoms of depression of any severity were detected, after discharge from the hospital three months, six months, 12 months after a heart attack. myocardium (MI); after establishing the presence of a depressive disorder, the use of the scales was carried out in the above terms. Patients were not included in the study group if the symptoms of depression were not detected for 3 months after the ischemic attack. 
According to ICD-10, a clinically significant depressive disorder consists of the following symptoms: main - a) a decrease in mood compared to the patient's normal norm, which occurs almost daily and most of the day, as well as regardless of the situation; b) a decrease (loss) of interests and the ability to experience pleasure in activities, usually associated with positive emotions; c) decreased activity, increased (pronounced) fatigue and decreased energy; and additional - a) sleep disorders of any type; b) a change in appetite (decrease or increase) with corresponding changes in body weight; c) recurring thoughts of death, suicide or self-harm; d) a gloomy and pessimistic vision of the future; e) ideas of guilt and selfdeprecation; f) decreased self-esteem and a sense of self-doubt; g) decreased concentration, inability to concentrate, decreased ability to think, indecision or hesitation in decision making. Mild depression ( $F 32.0$ according to ICD-10) is characterized by the presence of two main and two additional symptoms. Moderate depression (F 32.1 according to ICD-10) is characterized by the presence of two main and three to four additional symptoms. Severe depression ( $F$ 32.2 or $F \quad 32.3$ according to ICD-10) is characterized by the presence of three main, four or more additional symptoms, some of which are significant.

\section{RESEARCH RESULTS}

Among patients with $\mathrm{MI}$ with DS in the first days after $\mathrm{MI}$, more than half (51.1\%) reported a permanent decrease in mood more often than other symptoms, i.e. these patients showed an affective component of depression, while the ideator component of depression, which manifested itself in thinking retardation to one degree or another, was in $27.5 \%$ of patients, motor retardation (motor component) was detected in $21.5 \%$ of those observed. In patients with almost the same frequency, the anxious and melancholic type of affect was noted (47.0\% and $41.2 \%$, respectively), the dysphoric type of affect was found in $11.8 \%$ of cases, which is 4 times less than anxious and 3.5 times less than the melancholic type.

In accordance with the objectives of the study, the patient's condition was analyzed one month, three, six and twelve months after myocardial infarction. The same analysis was carried out in patients of the control group. It turned out that 1 month after myocardial infarction, only the control group had no depressive disorders, while the main group of patients had depressive syndromes. Clinically, $43.8 \%$ of patients had asthenic depression, $32.2 \%$ of DS were classified as anxious depression, $13.7 \%$ - melancholic, 10.3\% dysphoric depression. There is a noticeable change in the clinical vector of DS towards an increase in asthenic and a decrease in melancholic and anxiety depressions, which is obviously associated with an improvement in the somatic state of patients and, associated with this, a more adequate attitude to their condition and disease. This is confirmed by the data of the examination of patients three months after the MI.

In the main group, there is a noticeable reduction in symptoms in patients with melancholic and dysphoric depression, but an increase in the number of patients with anxiety depressive states. Interestingly, 18 patients (16.7\%) with anxiety depression appeared in the control group 3 months after myocardial infarction. Such a change occurred due to the need for certain restrictions in the patient's life: adherence to the work and rest regimen and the therapy regimen, rejection of the usual way of life and bad habits (smoking, alcohol), 
overprotection from loved ones unusual for the patient, reassessment of their social roles. It is necessary to pay attention to this group of patients, the clinical expression of depression in whom was: disturbance of falling asleep, superficial sleep, a feeling of fear of a recurrence of myocardial infarction, constant ideas of self-accusation of improper behavior that led to myocardial infarction, episodes of vegetative paroxysms with pronounced heartbeat, feeling of lack of air, unpleasant sensations in the area of the heart, which patients took for another $\mathrm{MI}$, called an ambulance, believed that they were examined by incompetent specialists, argued with doctors and relatives. However, according to the Hamilton and Montgomery-Asberg scales, these DSs were qualified as mild depressions, which required more psychotherapeutic intervention in the form of convincing patients that there were no MI symptoms and sleep correction.

6 months after MI, the number of patients without DR increased significantly. It turned out that with a significant decrease in patients with anxiety and asthenic depression, there appeared patients with a hypochondriac variant of DS, which was characterized by a constant search for symptoms of myocardial infarction, frequent measurement of pulse and blood pressure, visiting cardiologists and therapists, with frequent changes of doctors and distrust of consultations. In addition, these patients changed their attitude towards others; they demanded the attention of their relatives and tried to show everyone how seriously ill they were, practically stopped working and were passive to family problems. In the control group, the number of patients with anxious depression decreased by more than 2 times.
A somewhat different picture is observed one year after the MI. Among the studied patients, $7.3 \%$ of the main group and $0.9 \%$ of the control group retained symptoms of depression. Among DS in the main group, anxious and hypochondriac depressions were noted. In terms of severity, these depressions were characterized as mild. Awareness of the presence of a serious illness, fear of a recurrence of myocardial infarction, the inability to internally change the attitude to the transferred, lack of objective information about the disease and its consequences creates the basis for maintaining an internal psychological imbalance, which disturbs the patient's emotional response, as a result of which depressive disorders persist. Obviously, this group of patients requires close attention from cardiologists and psychiatrists with the development of a plan for psycho-corrective influence.

\section{DISCUSSIONS}

In cases of prolonged depression, the severity of affective disorders more often directly correlated with the severity of the physical condition. If not so long-term depressive episodes were largely due to "their own vision of the disease", the severity of their symptoms depended on the conversation with the doctor and the information received from him, the degree of awareness of his diagnosis, possible complications, and not very much depended on the general somatic status itself. Then the course of prolonged depression in patients worsened with the aggravation of the cardiological and general somatic condition of patients. Psychosomatic parallelism in the majority of protracted depressions was manifested by the generalization of asthenic symptom complexes (increased general weakness, intolerance to exertion, lethargy, 
adynamia, severe daytime sleepiness in combination with early insomnia) with deterioration of the somatic state. At the same time, it was noted that with prolonged depression, there were always more or less pronounced cognitive disorders (reduced memory for past events, limited ability to comprehend what was happening around, remember new information, impaired concentration).

In the foreground, with shorter depressive episodes, depression, a pessimistic perception of the disease, an exaggerated assessment of its consequences, anxious fears of a second attack of angina pectoris, fear of death, and imminent disability manifested themselves.

Heightened self-observation with the registration of the slightest changes in wellbeing was combined with a mass of complaints, sometimes without sufficient somatic justification. A significant place among other components of depressive disorder was occupied by hysterical (lump in the throat, tremor, numbness of the limbs in the form of "gloves"), somatovegetative (tachycardia, dyspnoea, insomnia) and asthenic (increased exhaustion, decreased activity, complaints of weakness, loss of activity) symptom complexes ... The reverse development of such depressions coincided with the period of stabilization of the manifestations of somatic illness, when the danger of death was minimized, motor activity began to recover, and the level of anxiety decreased.

The foregoing suggests that the duration of a depressive episode after an acute myocardial infarction may indicate the origin of this symptom complex. So, depressive episodes lasting up to six months can be attributed to nosogenies, that is, states that are the psyche's response to a traumatic situation, in our case, a serious somatic illness. Depressive episodes with a protracted course, probably, with a high degree of confidence can be attributed to somatogenies, that is, conditions pathophysiologically associated with severe pathology of internal organs, i.e. in patients with more severe forms of cardiac pathology, depression reduction occurs at a slower pace.

\section{CONCLUSIONS}

1. The vast majority of patients with acute myocardial infarction experience a depressive episode of moderate (moderate) severity at the initial diagnosis (71.6\% on the Hamilton scale and $72.7 \%$ on the Montgomery-Asberg scale);

2. Identification of symptoms of depression within a year after MI, even in patients of the control group, dictates the need to study the complex of factors that led to the formation of depressive disorders in patients with MI.

3. Postinfarction depressive episodes lasting up to six months can be attributed to nosogenies; depressive episodes of postinfarction genesis with a protracted course, probably with a high degree of confidence, can be attributed to somatogenias.

\section{REFERENCES}

1. Drobizhev M.Yu. Treatment of depression in the general somatic network // Psychiatry and psychotherapy.-M., 2003.T.5, No. 5.-P.8-12.

2. Krasnov V.N. Psychiatric disorders in general medical practice // Rus. honey. zhurn. - M., 2001.-T.9, N 25.-P.1187-1191. 
3. Smulevich $A B$ Depression in general medicine. - $M$ : Medical Information Agency, 2001. - $128 \mathrm{p}$.

4. Dickens C. M., McGowan I., Percival C. et al. Lack of a close confidant, but not depression, predicts further cardiac events after myocardial infarction // Heart. -2004.Vol.90.-P.518-522.

5. Beckers T. Symptoms of depression, acute myocardial infarction and total mortality in a community sample. Vision Res. 2001; 411: 417: 2297-2303.

6. Polyakova E.V. "Features of the formation of intrinsic motivation for therapy in patients with cardiovascular pathology", Ph.D. abstract, Veliky Novgorod, 2010.

7. Mukhamadieva N.B. Evaluation of the biochemical indicators of blood of patients after myocardial infarction with depressive syndrome. Central asian journal of pediatrics, 20192 (2): 111-117.

8. Penninx BW, Geerlings SW, Deeg DJ, van Eijk JT, van Tilburg W, Beekman AT: Minor and major depression and the risk of death in older persons. Arch Gen Psychiatry 56: 889-895,1999.

9. Muxamadiyeva N.B., Tuksanova Z.I. Integrated assessment of risk factors of post-infarction depressive disorders // Central Asian journal of Pediatrics. -2019. P. 101-111.

10. Muxamadiyeva N.B. Influence of depression on social characteristics of patients with myocardial infarction // European Journal of Biomedical and Pharmaceutical Sciences. - 2020. Volume 7. Issue 7. - P. 93-95.

11. Khodzhaev A.I. Dynamics of anxietydepressive disorders in patients with essential hypertension with cerebrovascular complications against the background of standard antihypertensive therapy // Bulletin of the Association of Physicians of Uzbekistan. - Tashkent, 2011. N3. - S. 26-28.

12. Muxamadiyeva N.B., Muxtorova H.K. Factors of formation of depressive spectrum disorders in patients who have suffered a myocardial infarction // European journal of pharmaceutical and medical research. - 2020. Volume 7-7. - P. 187-190. 